\title{
Emergency Physician-Performed Bedside Ultrasound of Gastric Volvulus
}

\author{
Keyon Shokraneh ${ }^{1}$, Jordan Johnson ${ }^{1}$, Gabriel Cabrera ${ }^{1}$, Eric J. Kalivoda ${ }^{1}$ \\ 1. Emergency Medicine, Hospital Corporation of America Healthcare West Florida Graduate Medical Education \\ Consortium/University of South Florida Morsani College of Medicine, Brandon Regional Hospital, Brandon, USA
}

Corresponding author: Eric J. Kalivoda, eric.j.kalivoda@gmail.com

\begin{abstract}
Gastric volvulus (GV) is a seldomly encountered life-threatening condition that necessitates rapid diagnosis in the emergency department (ED). The diagnosis of GV is traditionally established with cross-sectional imaging and/or endoscopy with surgical confirmation. The potential role of point-of-care ultrasound (POCUS) as a bedside tool to support the early identification of GV by emergency physicians (EPs) has not been thoroughly investigated. This case report describes the expeditious diagnosis and ED management of acute GV by implementing EP-performed POCUS into critical decision making.
\end{abstract}

Categories: Emergency Medicine, General Surgery

Keywords: point-of-care ultrasound, ultrasonography, gastric volvulus, emergency department

\section{Introduction}

Gastric volvulus (GV) is a rare surgical emergency with mortality rates approaching $30 \%-50 \%$ when complications occur [1-4]. The pathophysiology of GV is classified by an abnormal rotation of the stomach along the longitudinal (organoaxial) axis, transverse (mesenteroaxial) axis, or a combined axis. Gastric strangulation and complications of necrosis, perforation, and/or septic shock can result when rotation exceeds $180^{\circ}$ with complete closed-loop obstruction. Primary GV is usually attributed to neoplasm, adhesions, or abnormal stomach attachments, whereas paraesophageal hernia is the most common etiology of secondary GV in adults $[2,4]$.

Timely recognition and diagnosis of GV is imperative to avoid detrimental delays in the emergency department (ED) intervention and definitive management. Plain radiography and CT are the initial diagnostic modalities typically employed when GV is suspected; however, the utilization of ultrasonography to support the diagnosis has not been systematically studied [5-11]. The use of point-of-care ultrasound (POCUS) to diagnose acute abdominal emergencies, such as small bowel obstruction, is within the scope of practice for emergency physicians (EPs) $[12,13]$. Similarly, the identification of acute gastric outlet obstruction (GOO) (secondary to underlying malignancies and peptic ulcer disease) with POCUS has been described in two previous case reports $[14,15]$. To the best of our knowledge, this is the first case report describing EP-performed POCUS of GV secondary to paraesophageal hernia.

Received 08/17/2020 Review began 08/17/2020
Review ended 08/19/2020 Published 08/22/2020

\section{() Copyright 2020}

Shokraneh et al. This is an open access article distributed under the terms of the Creative Commons Attribution License CC-BY 4.0., which permits unrestricted use, distribution, and reproduction in any medium, provided the original author and source are credited.

\section{Case Presentation}

A 69-year-old male with a past medical history of hypertension, atrial fibrillation, antineutrophil cytoplasmic antibody (ANCA) vasculitis, chronic kidney disease, gastroesophageal reflux disease, and hiatal hernia presented to the ED with chief complaint of abdominal pain. He described a one-day history of gradual-onset, constant, dull, moderate severity, non-radiating epigastric discomfort with associated abdominal distention, nausea, and decreased appetite. He reported two episodes of vomiting prior to arrival. He denied fevers, chills, diarrhea, constipation, dark or bloody stools, flank pain, back pain, urinary complaints, chest pain, shortness of breath, palpitations, or syncope. Initial vital signs were temperature $36.8^{\circ} \mathrm{C}$, blood pressure $157 / 106 \mathrm{mmHg}$, heart rate 83 beats per minute, respiratory rate 14 breaths per minute, and oxygen saturation of $95 \%$ on room air. Physical examination revealed an elderly male in moderate distress secondary to pain. Significant tenderness to palpation in the epigastrium and left upper quadrant was elicited on abdominal examination without rebound, guarding, or peritoneal signs. The remainder of the physical examination was unremarkable. Electrocardiogram (ECG) revealed normal sinus rhythm without acute ischemic changes.

The clinical presentation was concerning for an acute intra-abdominal emergency. CT of the abdomen and pelvis, laboratory studies, pain medications, antiemetics, and intravenous (IV) fluids were ordered. Emergency medicine resident physicians and an ultrasound fellowship-trained attending EP then performed an abdominal POCUS examination. POCUS was notable for a large, dilated, fluid-filled stomach highly concerning for an obstructive process (Figures 1, 2; Videos 1,2). 


\section{Cureus}

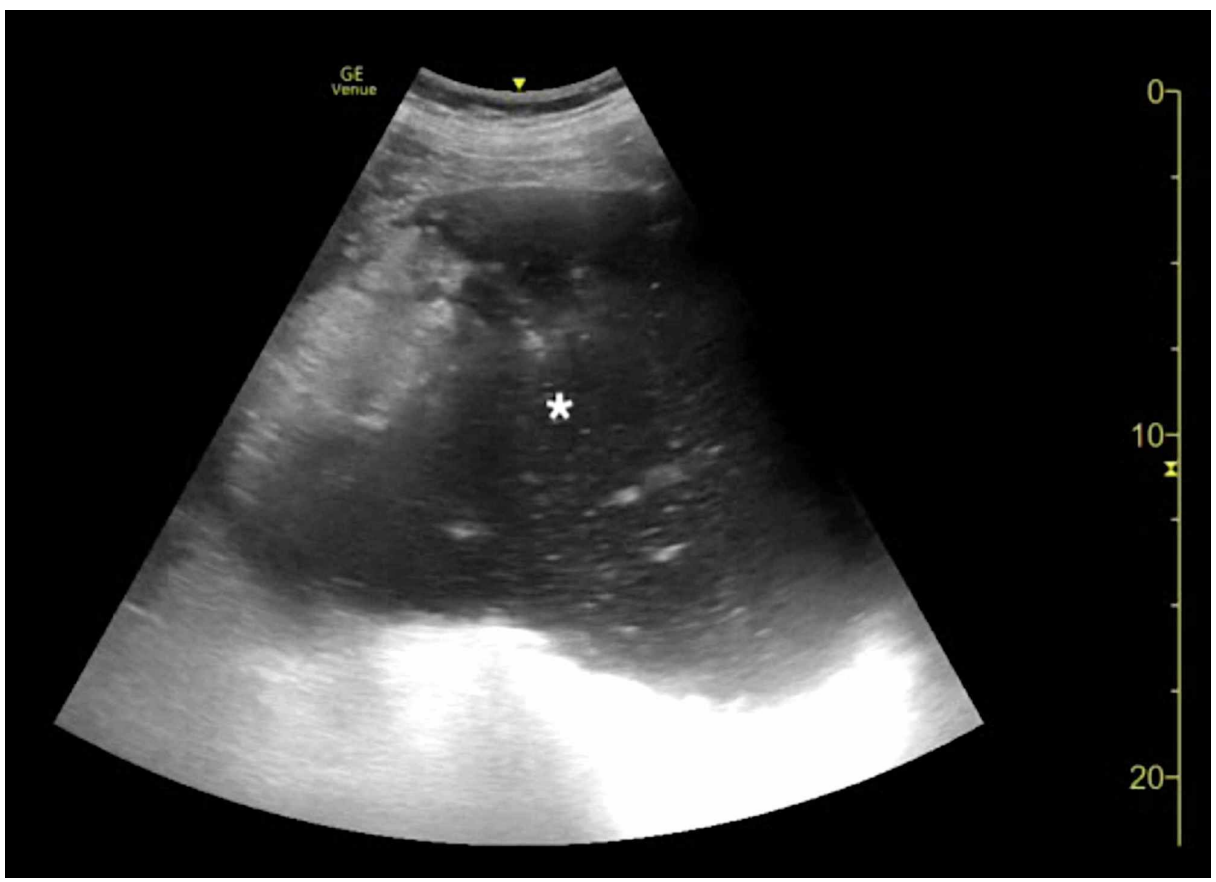

FIGURE 1: Point-of-care ultrasound of gastric volvulus demonstrating a distended, fluid-filled stomach (asterisk).

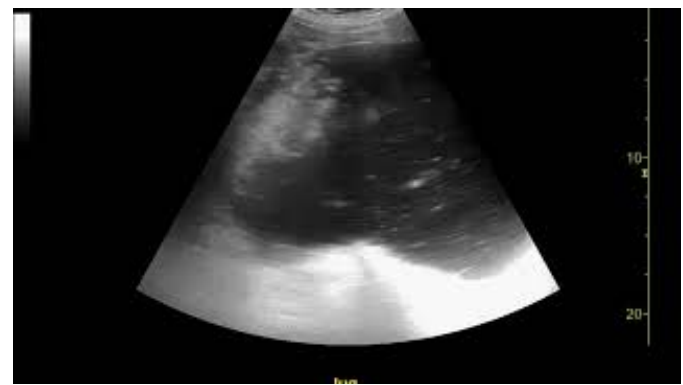

VIDEO 1: Point-of-care ultrasound of gastric volvulus demonstrating a distended, fluid-filled stomach.

View video here: https://www.youtube.com/watch?v=qsyaUq-y5BY\&feature=youtu.be 


\section{Cureus}

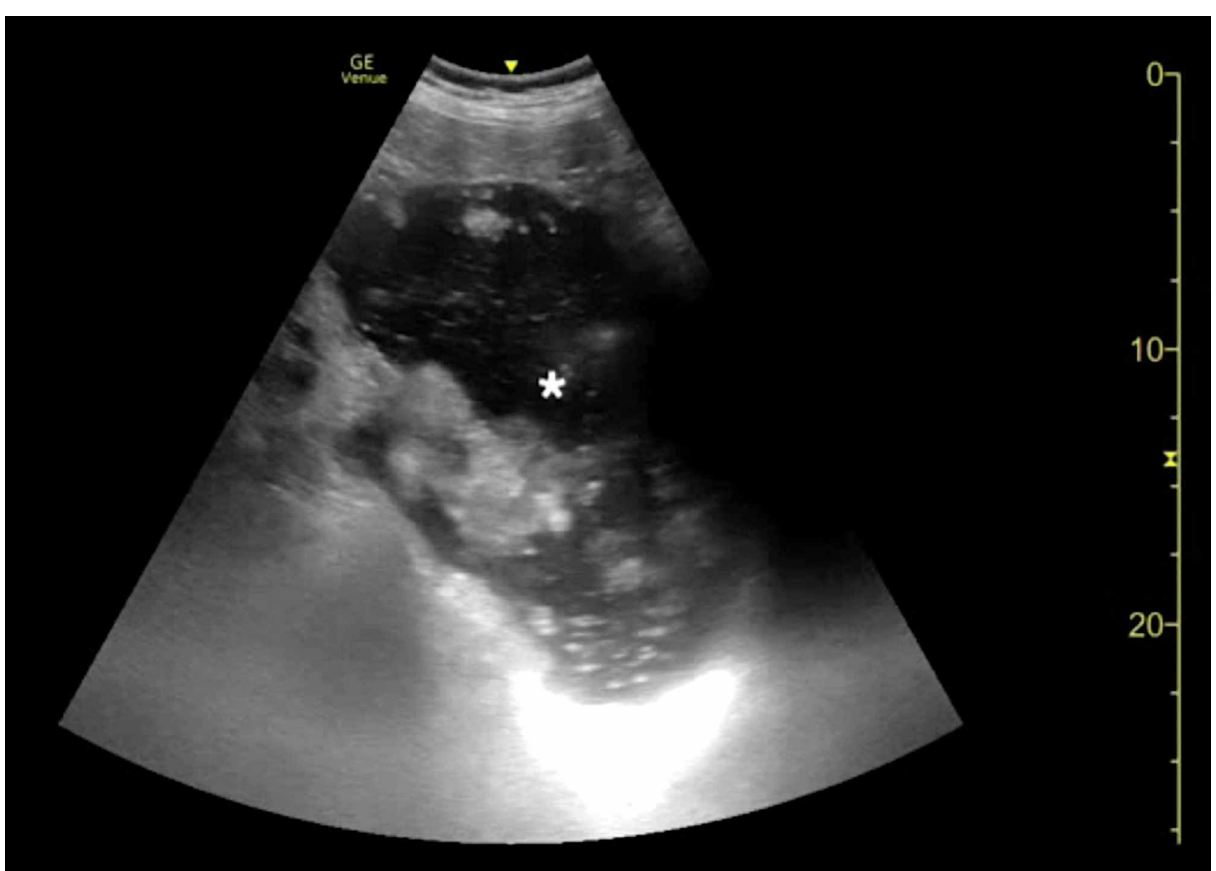

FIGURE 2: Point-of-care ultrasound of gastric volvulus demonstrating a distended, fluid-filled stomach (asterisk).

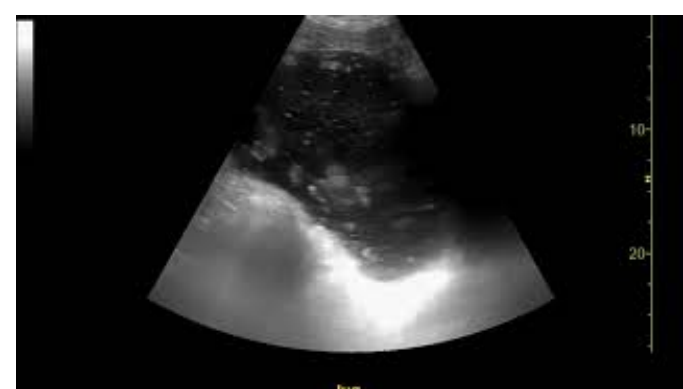

VIDEO 2: Point-of-care ultrasound of gastric volvulus demonstrating a distended, fluid-filled stomach.

View video here: https://www.youtube.com/watch?v=X2kc8w-ITp0\&feature=youtu.be

CT imaging was subsequently expedited based upon the POCUS findings. CT of the abdomen/pelvis revealed a moderate to large hiatal hernia and a distended edematous stomach with partial obstruction due to GV (Figures 3-5). General surgery was consulted emergently and evaluated the patient at bedside in the ED. Multiple prolonged attempts at nasogastric tube (NGT) insertion were initially unsuccessful. NGT placement was ultimately successful yielding $\sim 2,000 \mathrm{~mL}$ of non-bilious stomach contents, and repeat POCUS confirmed gastric decompression (Figure 6). Laboratory analysis was unremarkable for acute clinically significant abnormalities. 


\section{Cureus}

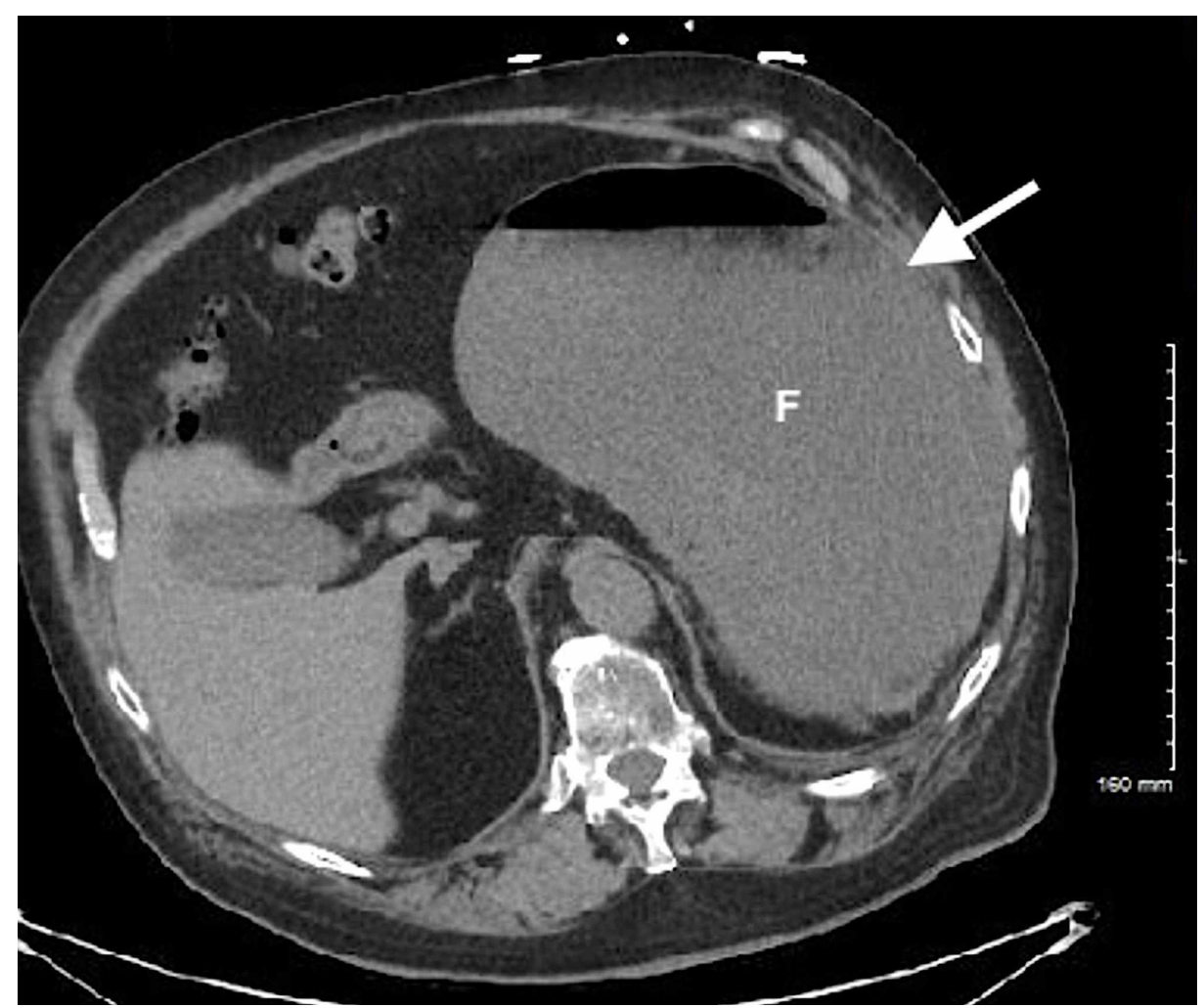

FIGURE 3: CT of the abdomen/pelvis (axial view) demonstrating gastric volvulus with a fluid-filled distended stomach (solid arrow).

F, gastric fundus.

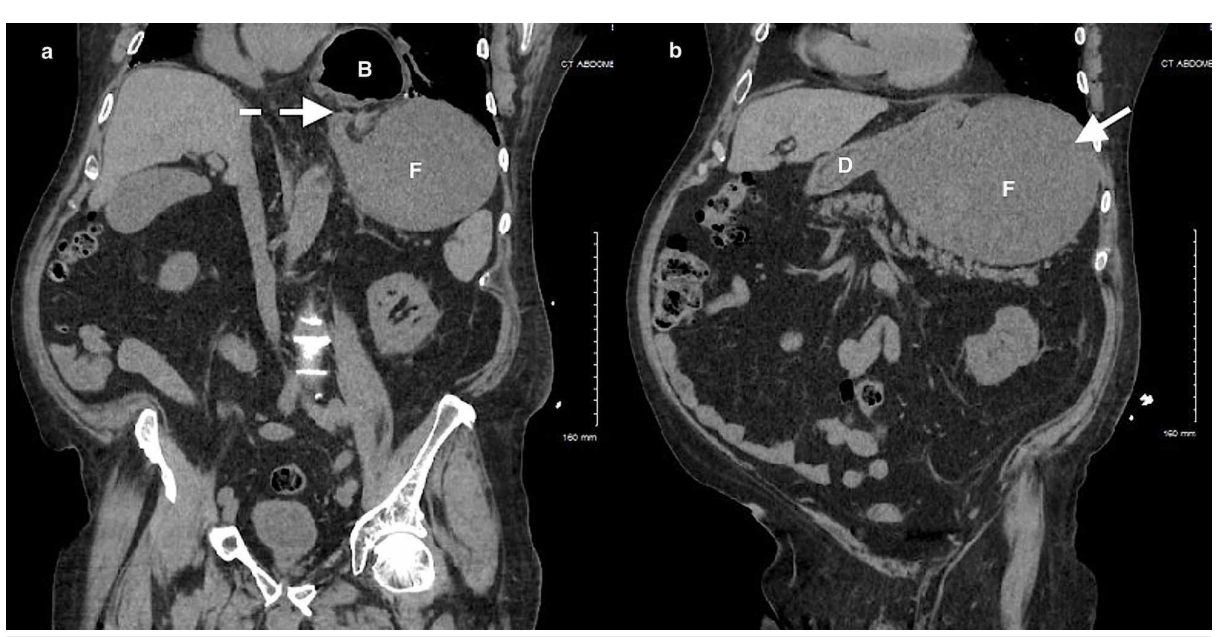

FIGURE 4: CT of the abdomen/pelvis (coronal views) demonstrating gastric volvulus with (a) malrotation (dotted arrow) and a (b) fluid-filled distended stomach (solid arrow).

B, gastric body; F, gastric fundus; D, duodenum. 


\section{Cureus}

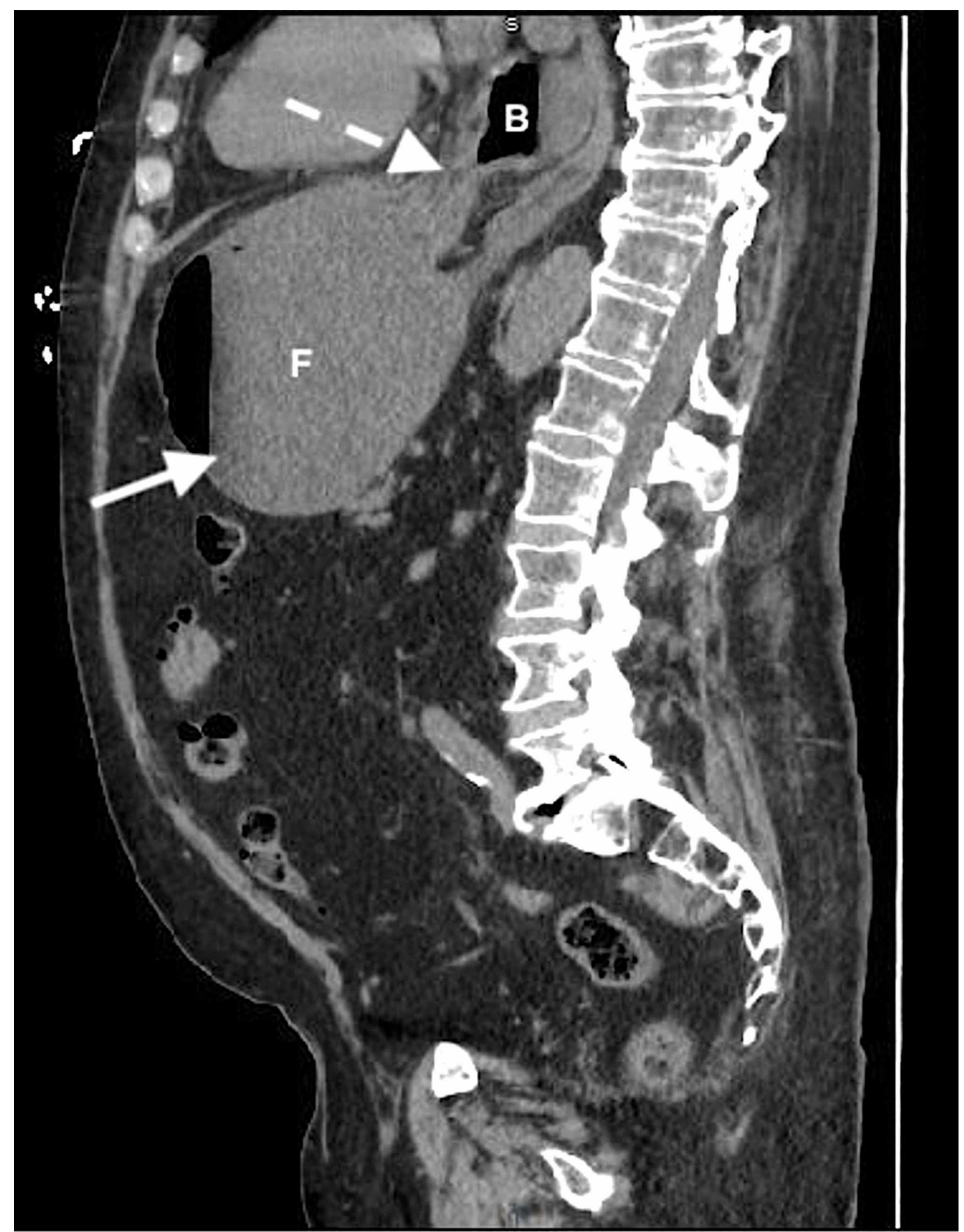

FIGURE 5: CT of the abdomen/pelvis (sagittal view) demonstrating gastric volvulus with a fluid-filled distended stomach (solid arrow) and malrotation (dotted arrow).

B, gastric body; F, gastric fundus. 


\section{Cureus}

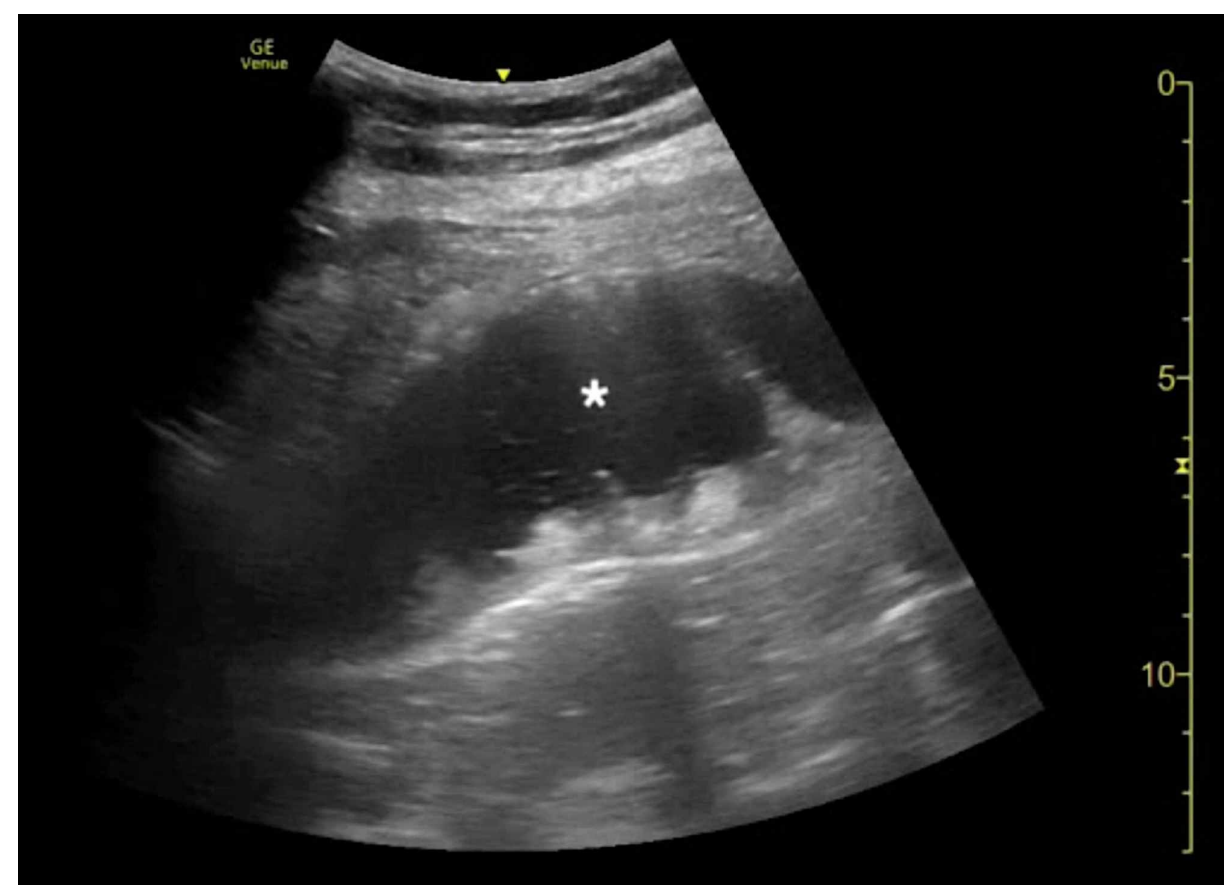

\section{FIGURE 6: Point-of-care ultrasound of gastric volvulus post-nasogastric tube insertion demonstrating stomach decompression (asterisk).}

The patient was admitted for further management of GV. Gastroenterology was consulted for emergent esophagogastroduodenoscopy (EGD) that confirmed the presence of a partial organoaxial volvulus without evidence of gastric ischemia. EGD did not reveal any underlying pathology in the upper gastrointestinal tract with the exception of large hiatal hernia and mild gastritis. General surgery then performed laparoscopic reduction of GV, hiatal hernia repair with mesh, and Nissen fundoplication. The patient was soon tolerating a full liquid diet and closely managed post-operatively by general surgery and gastroenterology. The patient was discharged home in stable condition on hospital day 6 with instructions for outpatient surgery follow-up.

\section{Discussion}

The ED evaluation of acute abdominal pain requires a diligent and meticulous approach to identify critical underlying pathology. Symptoms may be vague or non-specific, and the classic constellation of clinical findings may not be present. Acute GV with complete obstruction will oftentimes be recognized with the classical Borchardt's triad of intractable retching, severe epigastric abdominal pain and distention, and difficulty or inability to pass an NGT [2,4]. Importantly, GV can mimic other common causes of epigastric pain, such as gastroesophageal reflux disease or peptic ulcer disease, and may even present with complaint of chest pain [16,17]. Our case patient notably did report epigastric pain, but did not experience any further intractable retching or vomiting during his ED course; we did experience significant difficulty with NGT insertion before it was eventually placed, suggesting an incomplete obstructive process. GV can be a challenging diagnosis in the ED setting; therefore, EPs should maintain a higher degree of clinical suspicion in patients with a known underlying hiatal hernia.

The choice of initial imaging study is significant when there is suspicion for GV, as diagnostic delay can lead to fatal complications. CT imaging is highly reliable and accurate, with two findings (a normal gastropyloric transition zone and the antrum in an abnormal location) with $100 \%$ sensitivity and specificity for the diagnosis of acute GV $[18,19]$. One retrospective study investigated radiologists' accuracy of CT interpretation for GV, with reported $90 \%$ overall accuracy [19]. Interestingly, radiologists were able to make the critical distinction between GV and large hiatal hernia, which are notoriously difficult entities to distinguish, and especially notable because most GV cases are associated with paraesophageal hernias.

POCUS is an effective non-invasive imaging modality for the bedside assessment of a multitude of abdominal emergencies. Moreover, POCUS offers the vital advantages of avoiding radiation exposure and is readily available for repeat serial examinations as dictated by the clinical scenario. Previous literature has reported the utilization of POCUS for identifying gastric dilation in cases of GOO [14,15]. We discerned comparable sonographic findings in our case, with visualization of a severely dilated stomach and layering of gastric contents similar to the "black-and-white cookie" sign of GOO [15]. We were also able to use POCUS to dynamically observe successful gastric decompression. EPs should consider utilizing bedside ultrasonography for the rapid verification of appropriate NGT placement. This case highlights another 


\section{Conclusions}

GV is a life-threatening surgical emergency that warrants prompt EP recognition and management. Implementation of POCUS findings into clinical decision making has the potential to facilitate an expedited diagnosis. Further larger-scale studies should investigate the diagnostic accuracy and ideal role of EPperformed POCUS as a first-line imaging strategy for GV.

\section{Additional Information \\ Disclosures}

Human subjects: Consent was obtained by all participants in this study. issued approval \#1635. HCA Healthcare West Florida Graduate Medical Education Division Research Director issued approval \#1635. Conflicts of interest: In compliance with the ICMJE uniform disclosure form, all authors declare the following: Payment/services info: All authors have declared that no financial support was received from any organization for the submitted work. Financial relationships: All authors have declared that they have no financial relationships at present or within the previous three years with any organizations that might have an interest in the submitted work. Other relationships: All authors have declared that there are no other relationships or activities that could appear to have influenced the submitted work.

\section{Acknowledgements}

This research was supported (in whole or in part) by HCA and/or an HCA affiliated entity. The views expressed in this publication represent those of the author(s) and do not necessarily represent the official views of HCA or any other affiliated entities.

\section{References}

1. Teague WJ, Ackroyd R, Watson DI, Devitt PG: Changing patterns in the management of gastric volvulus over 14 years. Br J Surg. 2000, 87:358-361. 10.1046/j.1365-2168.2000.01385.x

2. Rashid F, Thangarajah T, Mulvey D, Larvin M, Iftikhar SY: A review article on gastric volvulus: a challenge to diagnosis and management. Int J Surg. 2010, 8:18-24. 10.1016/j.ijsu.2009.11.002

3. Light D, Links D, Griffin M: The threatened stomach: management of the acute gastric volvulus . Surg Endosc. 2016, 30:1847-1852. 10.1007/s00464-015-4425-1

4. Bauman ZM, Evans CH: Volvulus. Surg Clin North Am. 2018, 98:973-993. 10.1016/j.suc.2018.06.005

5. Godshall D, Mossallam U, Rosenbaum R: Gastric volvulus: case report and review of the literature . J Emerg Med. 1999, 17:837-840. 10.1016/s0736-4679(99)00092-X

6. Tanen DA: Acute gastric volvulus: a case report . Acad Emerg Med. 1999, 6:83-84. 10.1111/j.15532712.1999.tb00100.x

7. Chau B, Dufel S: Gastric volvulus. Emerg Med J. 2007, 24:446-447. 10.1136/emj.2006.041947

8. Wu MH, Chang YC, Wu CH, Kang SC, Kuan JT: Acute gastric volvulus: a rare but real surgical emergency . Am J Emerg Med. 2010, 28:118.e5-118.e7. 10.1016/j.ajem.2009.04.031

9. Kiyani A, Khosla M, Anufreichik V, Chuang KY: “A large hiatal hernia”: atypical presentation of gastric volvulus. Clin Pract Cases Emerg Med. 2017, 1:187-189. 10.5811/cpcem.2017.2.31075

10. Simon E, Cohen J, Long B: Male with sudden onset abdominal pain . J Emerg Med. 2017, 53:e109-e110. 10.1016/j.jemermed.2017.08.001

11. Patel AV, Senatore FJ, Bhurwal A: Epigastric pain due to acute gastric volvulus . J Emerg Med. 2019, 57:185186. 10.1016/j.jemermed.2019.09.010

12. Lewiss RE, Pearl M, Nomura JT, et al.: CORD-AEUS: consensus document for the emergency ultrasound milestone project. Acad Emerg Med. 2013, 20:740-745. 10.1111/acem.12164

13. Gottlieb M, Peksa GD, Pandurangadu AV, Nakitende D, Takhar S, Seethala RR: Utilization of ultrasound for the evaluation of small bowel obstruction: a systematic review and meta-analysis. Am J Emerg Med. 2018, 36:234-242. 10.1016/j.ajem.2017.07.085

14. Gottlieb M, Nakitende D: Identification of gastric outlet obstruction using point-of-care ultrasound. Am J Emerg Med. 2017, 35:1207.e1-1207.e2. 10.1016/j.ajem.2017.03.024

15. Cohen A, Foster M, Stankard B, Owusu M, Nelson M: The "Black-and-White Cookie" sign- a case series of a novel ultrasonographic sign in gastric outlet obstruction. Clin Pract Cases Emerg Med. 2018, 2:21-25. 10.5811/cpcem.2017.11.35890

16. Porter DN, Emerson C, Ward P: The best way to a woman's heart? . Emerg Med J. 2011, 28:247-248. 10.1136/emj.2009.089078

17. Ghosh RK, Fatima K, Ravakhah K, Hassan C: Gastric volvulus: an easily missed diagnosis of chest pain in the emergency room. BMJ Case Rep. 2016, 2016:bcr2015213888. 10.1136/bcr-2015-213888

18. Millet I, Orliac C, Alili C, Guillon F, Taourel P: Computed tomography findings of acute gastric volvulus . Eur Radiol. 2014, 24:3115-3122. 10.1007/s00330-014-3319-2

19. Mazaheri P, Ballard DH, Neal KA, Raptis DA, Shetty AS, Raptis CA, Mellnick VM: CT of gastric volvulus: interobserver reliability, radiologists' accuracy, and imaging findings. AJR Am J Roentgenol. 2019, 212:103108. 10.2214/AJR.18.20033 